\title{
ESTUDO DA DISTRIBUIÇÃO DE FASES Fe-Zn EM REVESTIMENTOS GALVANNEALED UTILIZANDO A TÉCNICA GDOES
}

\author{
Sandra Goulart-Santos ' \\ Deyselane de Fátima Anício ${ }^{2}$ \\ Aldo Henrique de Almeida Barbosa ${ }^{3}$
}

\section{Resumo}

Os revestimentos galvannealed (GA) são constituídos por fases Fe-Zn, sendo sua qualidade bastante afetada pela distribuição dessas fases, tornando-se importante caracterizá-la. A Espectroscopia de Emissão Ótica por Descarga Luminescente (GDOES) é uma técnica que permite avaliar o perfil de composição química ao longo da espessura de forma rápida e precisa. Neste estudo, foram produzidos revestimentos $\mathrm{GA}$ variando-se as temperaturas $\left(530^{\circ} \mathrm{C}, 550^{\circ} \mathrm{C}, 580^{\circ} \mathrm{C}\right)$ e os tempos (0 s, $5 \mathrm{~s}$, $15 \mathrm{~s}, 60 \mathrm{~s}$ ) de tratamento de galvanneling, de modo a se alterar a distribuição das fases Fe-Zn. Os revestimentos foram caracterizados por MEV e DRX. Análises de GDOES foram realizadas visando identificar as distribuições de Fe e Al e correlacioná-las com as fases $\eta, \zeta, \delta$ e $\Gamma$. Verificou-se que as variações de temperatura e o tempo de tratamento influenciaram na distribuição de Fe e Al. Para tempos baixos, Fe e Al se concentraram próximo à interface revestimento/substrato e com o aumento do tempo as concentrações se tornaram mais uniformes. A caracterização dos revestimentos GA a partir da distribuição de Fe e Al se mostrou eficaz para a verificação da distribuição das fases Fe-Zn. Palavras-chave: Revestimentos; Galvanneal; Fases Fe-Zn; Espectroscopia de emissão ótica por descarga luminescente.

\section{STUDY OF THE Fe-Zn PHASES DISTRIBUITON ON GALVANNELED COATINGS USING GDOES}

\begin{abstract}
Galvannealed (GA) coatings are composed of Fe-Zn phases, being its quality greatly affected by the phase's distribution, making it important to be characterized. Glow Discharge Optical Emission Spectroscopy (GDOES) technique allows a quick and accurate evaluation of the chemical composition profile along the coating thickness. In this study, GA coatings were produced varying the temperature $\left(530^{\circ} \mathrm{C}, 550^{\circ} \mathrm{C}, 580^{\circ} \mathrm{C}\right)$ and times $(0 \mathrm{~s}, 5 \mathrm{~s}, 15 \mathrm{~s}, 60 \mathrm{~s})$ of galvannealing treatment, in order to change the Fe-Zn phases distribution. The coatings were characterized by SEM and XRD. GDOES analyses were performed to identify the distributions of $\mathrm{Fe}$ and $\mathrm{Al}$ and correlate them with the $\eta, \zeta, \delta$ and $\Gamma$ phases. It was found that variations in temperature and treatment time influenced the distribution of Fe and Al. For short times, $\mathrm{Fe}$ and $\mathrm{Al}$ were concentrated near the coating/substrate interface and as the time increase concentrations became more uniform along the coating. The characterization of GA coatings from the Fe and Al distribution were effective for checking the distribution of $\mathrm{Zn}-\mathrm{Fe}$ phases.
\end{abstract}

Key words: Coatings; Galvanneal; Fe-Zn phases; Glow discharge optical emission spectroscopy.

\footnotetext{
'Engenheira Química, Doutora em Engenharia Metalúrgica e de Minas, Pesquisadora Plena, Centro de Tecnologia Usiminas, Av. Pedro Linhares Gomes, 543I, Cep 35I60-900, Ipatinga, MG, Brasil.E-mail: sandra.goulart@usiminas.com

${ }^{2}$ Técnica em Metalurgia, Técnica de Pesquisa, Centro de Tecnologia Usiminas, Av. Pedro Linhares Gomes, 543I, Cep 35I60-900, Ipatinga, MG,

Brasil.E-mail: deyselane.anicio@usiminas.com

${ }^{3}$ Engenheiro Metalurgista, Doutor em Engenharia Metalúrgica e de Minas, Pesquisador Especialista, Centro de Tecnologia Usiminas, Av. Pedro Linhares Gomes, 543I, Cep 35160-900, Ipatinga, MG, Brasil.E-mail: aldo.barbosa@usiminas.com
} 


\section{INTRODUÇÃO}

Os aços galvannealed (GA) são produzidos pelo processo de galvanização a quente, que consiste na imersão de tiras de aço em um banho de $\mathrm{Zn}$ contendo $\mathrm{Al} \mathrm{e} \mathrm{Fe}$, e posterior tratamento térmico do revestimento formado. Durante o tratamento térmico de galvannealing são formadas fases intermetálicas Fe-Zn, devido à difusão de Fe do substrato para a camada de $Z n$. Nesse processo podem ser formadas as seguintes fases: $\zeta\left(\mathrm{FeZn}_{13}\right), \delta$ $\left(F e Z n_{7}\right), \Gamma_{1}\left(F_{11} Z_{40}\right)$ e $\Gamma\left(F_{3} Z_{10}\right) \cdot(1,2) A$ presença de Al no banho de $Z n$ leva a formação da chamada camada inibidora na superfície da tira de aço. Normalmente, esta camada é formada por compostos Fe-Al e/ou Fe-Al-Zn, sendo a principal responsável pela incorporação do Al nos revestimentos $\mathrm{GA}$. (1)

A qualidade dos aços GA está diretamente relacionada com a distribuição das fases $\mathrm{Fe}-\mathrm{Zn}$ ao longo do revestimento, sendo a caracterização destas de grande relevância. Usualmente, a determinação das fases presentes nos revestimentos GA é realizada por microscopia e difração de raios $X$, mas outras técnicas podem ser utilizadas, dentre elas a Espectroscopia de Emissão Ótica por Descarga Luminescente (Glow Discharge Optical Emission Spectroscopy - GDOES).(3) A técnica GDOES realiza a análise química elementar da superfície de sólidos e também permite a determinação do perfil de concentração de elementos químicos ao longo da espessura do material (Quantitative Depth Profile-QDP). Durante a análise, a superfície é bombardeada continuamente por íons de argônio (sputtering), o que promove a remoção de camadas atômicas. Os átomos removidos são ionizados em um plasma e o espectro característico dos elementos medido pelo espectrômetro. ${ }^{(4)} A$ correlação entre a quantidade de material removido e o tempo de bombardeamento permite a determinação do perfil de composição da superfície, i.e., da curva concentração versus profundidade, normalmente expressas em termos de percentagem em peso (\%p) por micrometro. ${ }^{(5)}$

Neste trabalho, foi avaliada a aplicação da técnica GDOES no estudo da formação e da distribuição das fases $\mathrm{Fe}-\mathrm{Zn}$ em revestimentos GA, utilizando-se para tal os perfis de concentração de $\mathrm{Fe}$ e Al.

\section{MATERIAIS E MÉTODOS}

As amostras de revestimentos galvannealed foram produzidas em escala piloto utilizando um simulador do processo de galvanização a quente (HDPS - Hot Dip Process Simulator), tendo como substrato o aço IF. Foi utilizado um banho de zinco com teor de Al efetivo de aproximadamente $0,10 \%$ p. As condições de temperatura e tempo de encharque do tratamento térmico de galvannealing foram modificadas de modo a se alterar, propositalmente, a distribuição das fases $\mathrm{Fe}-\mathrm{Zn}$ no revestimento e são mostradas na Tabela I. Após o tempo de encharque, as amostras passaram por um processo de resfriamento rápido $\left(\sim 80^{\circ} \mathrm{C} / \mathrm{s}\right)$ com He para congelar a microestrutura dos revestimentos, possibilitando a análise do processo de formação das fases Fe-Zn.

Para avaliação da microestrutura, as seções dos revestimentos foram polidas, atacadas com Nital $0,2 \%$ e analisadas por microscopia eletrônica de varredura (MEV). Para identificação das fases cristalinas presentes nos revestimentos foram realizadas análises de difração de raios $X$ (DRX) na configuração $\theta-2 \theta$. As análises de GDOES foram realizadas no espectrômetro LECO GDS-850A com método de análise baseado na norma ISO 16962:2005,(6) que estabelece os critérios para análise química superficial de revestimentos metálicos a base de $\mathrm{Zn}$ e/ou Al. Foram avaliados os perfis de $\mathrm{Fe}, \mathrm{Al}$ e $\mathrm{Zn}$ (\%p) ao longo da espessura dos revestimentos. Os perfis de composição foram determinados em uma área de $4 \mathrm{~mm}$ de diâmetro. As distribuições das fases $\mathrm{Fe}-\mathrm{Zn}$ foram avaliadas tendo como referência as seguintes faixas de composição química: $\eta(<1 \% \mathrm{Fe}), \zeta(5,8-6,5 \% \mathrm{Fe}), \delta(7,3-\mathrm{II}, 7 \% \mathrm{Fe})$, $\Gamma_{1}(16,3-18,5 \% \mathrm{Fe})$ e $\Gamma(22,2-28,2 \% \mathrm{Fe})$. Estas faixas de composição foram comparadas com as microestruturas de seção dos revestimentos $\mathrm{GA}$, visando correlacionar os perfis de concentração de $\mathrm{Fe}$ e $\mathrm{Al}$ com as estruturas observadas durante o crescimento das fases Fe-Zn.

\section{RESULTADOS}

A caracterização dos revestimentos por MEV e DRX permitiu identificar os diferentes estágios de formação das fases $\mathrm{Fe}-\mathrm{Zn}$ que os revestimentos atingiram devido à variação de temperatura e tempo de encharque no tratamento de galvanneling. Visando acompanhar a evolução do processo de formação de fases $\mathrm{Fe}-\mathrm{Zn}$, para as temperaturas de $530^{\circ} \mathrm{C}, 550^{\circ} \mathrm{C}$ e $580^{\circ} \mathrm{C}$, optou-se por apresentar os resultados de microestrutura em função do tempo.

As microestruturas das seções dos revestimentos são mostradas na Figura I. Para os revestimentos processados sem encharque $(t=0 \mathrm{~s})$, observou-se a formação

Tabela I. Condições de temperatura e tempo de tratamento de galvannealing.

\begin{tabular}{|c|c|c|c|c|c|c|c|c|c|c|}
\hline \multirow{2}{*}{ Condição HDPS } & \multicolumn{10}{|c|}{ Identificação } \\
\hline & AI & $A 2$ & A3 & A4 & A5 & A6 & A7 & A8 & A9 & $A 10$ \\
\hline $\mathrm{T}\left({ }^{\circ} \mathrm{C}\right)$ & 530 & 530 & 530 & 550 & 550 & 550 & 550 & 580 & 580 & 580 \\
\hline Tempo (s) & 0 & 5 & 15 & 0 & 5 & 15 & 60 & 0 & 5 & 15 \\
\hline
\end{tabular}


de fases $\mathrm{Fe}-\mathrm{Zn}$ compactas até cerca de $50 \%$ da espessura para todas as temperaturas (Figuras la, Id e Ih). No entanto, para as temperaturas de $530^{\circ} \mathrm{C}$ e $550^{\circ} \mathrm{C}$ foram observadas regiões com o revestimento de $\mathrm{Zn}$ ainda não transformado (Figuras Ia, Id). Também foram observados cristais $\mathrm{Fe}-\mathrm{Zn}$ dispersos na matriz de $\mathrm{Zn}$ na região mais próxima a superfície, sendo que quanto maior a temperatura, maior quantidade desses cristais foi formada.

Para o tempo de encharque de cinco segundos, foi observado, para todas as temperaturas, que a formação de cristais das fases $\mathrm{Fe}-\mathrm{Zn}$ alcançou a superfície do revestimento e que a parte inferior da camada, próxima a interface do aço, tornou-se mais compacta (Figuras lb, le e li). Para os revestimentos processados a $550^{\circ} \mathrm{C}$ e $580^{\circ} \mathrm{C}$, foram observadas algumas cavidades próximas à superfície. Tais cavidades foram formadas pela dissolução do zinco presente nestas regiões durante $o$ ataque químico, indicando que a transformação do $\mathrm{Zn}$ em fases $\mathrm{Fe}-\mathrm{Zn}$ ainda estava incompleta nesse tempo de tratamento térmico. Observou-se também o aparecimento de uma fina camada na interface revestimento/substrato que pode ser associada à formação de uma fase $\mathrm{Fe}-\mathrm{Zn}$ com maior teor de ferro. Após $15 \mathrm{~s}$ de encharque, os revestimentos processados nas temperaturas de $550^{\circ} \mathrm{C}$ e $580^{\circ} \mathrm{C}$ apresentaram estrutura completamente transformada, isto é, todo $\circ \mathrm{Zn}$ inicial foi transformado em compostos intermetálicos $\mathrm{Fe}-\mathrm{Zn}$ (Figuras If e Ij). Apenas para a temperatura de $530^{\circ} \mathrm{C}$ foram observadas algumas cavidades próximas à superfície, indicativas de transformação $\mathrm{Fe}-\mathrm{Zn}$ incompleta (Figuras 2c). Observou-se para todos os revestimentos 0 crescimento da camada com maior teor de Fe na interface revestimento/substrato (Figuras Ic, If e Ij).

O tratamento térmico com tempo de encharque de $60 \mathrm{~s}$ foi realizado apenas na temperatura de $550^{\circ} \mathrm{C}$. Este revestimento apresentou estrutura completamente transformada em compósitos intermetálicos $\mathrm{Fe}-\mathrm{Zn}$, mostrando duas camadas bem definidas, sendo uma situada na interface revestimento/substrato associada à fase $\mathrm{Fe}-\mathrm{Zn}$ mais rica em ferro e outra superficial (Figura Ig).

As fases cristalinas dos revestimentos foram determinadas por difração de raios $X$. Para os revestimentos processados a $530^{\circ} \mathrm{C}$ foram identificadas as fases $\mathrm{Fe}-\mathrm{Zn} \zeta$, $\delta$ e $\Gamma_{1}$, e a fase $\eta$ associada ao zinco. Para a temperatura de $550^{\circ} \mathrm{C}$, foram identificadas as fases $\zeta, \delta$ e $\Gamma_{\text {, em todas as }}$ amostras. A fase $\eta$ foi detectada apenas nos revestimentos tratados durante $0 \mathrm{~s}$ e $5 \mathrm{~s}$, indicando que para esses tempos o zinco ainda não foi totalmente convertido em compostos Fe-Zn. Para a temperatura de $580^{\circ} \mathrm{C}$, as fases $\zeta, \delta$ e $\Gamma$, foram identificadas em todas as amostras e a fase $\eta$ não foi observada apenas para o tempo de $15 \mathrm{~s}$. Apesar

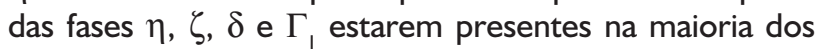
revestimentos avaliados, a intensidade dos picos e, consequentemente, as quantidades relativas de cada fase variou com as condições de tratamento térmico. Quanto maior o tempo de encharque e a temperatura, maior a intensidade dos picos das fases $\mathrm{Fe}-\mathrm{Zn}$ com teores mais elevados de $\mathrm{Fe}$, isto é, as fases $\delta$ e $\Gamma_{1}$.

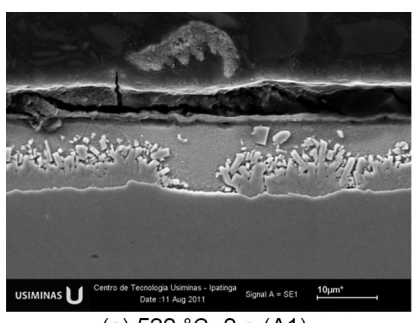

(a) $530{ }^{\circ} \mathrm{C}, 0 \mathrm{~s}(\mathrm{~A} 1)$

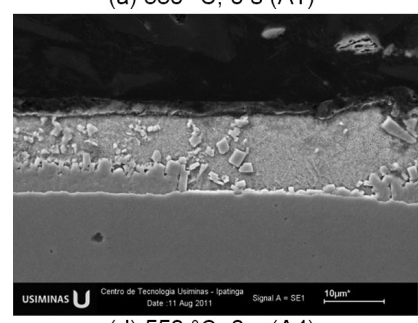

(d) $550^{\circ} \mathrm{C}, 0 \mathrm{~s}(\mathrm{~A} 4)$

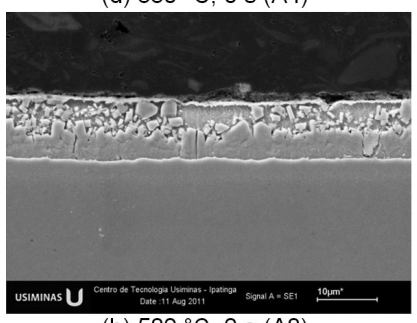

(h) $580{ }^{\circ} \mathrm{C}, 0 \mathrm{~s}(\mathrm{~A} 8)$

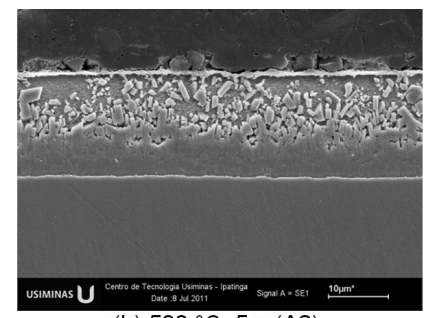

(b) $530^{\circ} \mathrm{C}, 5 \mathrm{~s}(\mathrm{~A} 2)$

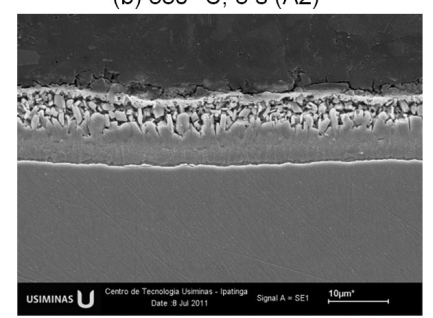

(e) $550^{\circ} \mathrm{C}, 5 \mathrm{~s}$ (A5)

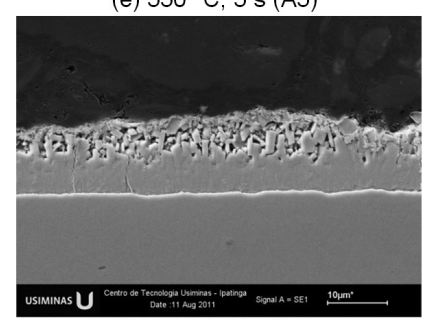

(i) $580^{\circ} \mathrm{C}, 5 \mathrm{~s}$ (A9)

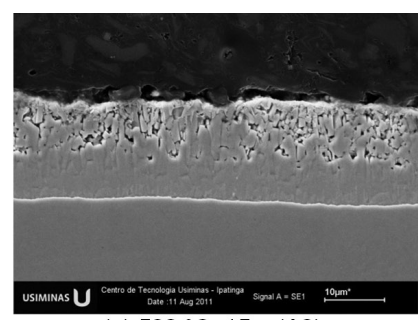

(c) $530^{\circ} \mathrm{C}, 15 \mathrm{~s}(\mathrm{~A} 3)$

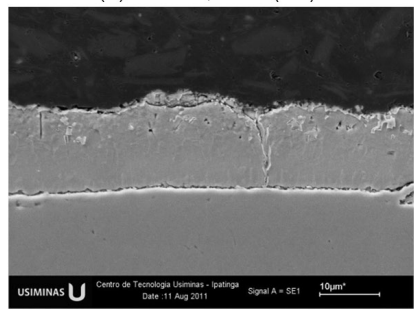

(f) $550{ }^{\circ} \mathrm{C}, 15 \mathrm{~s}(\mathrm{~A} 6)$

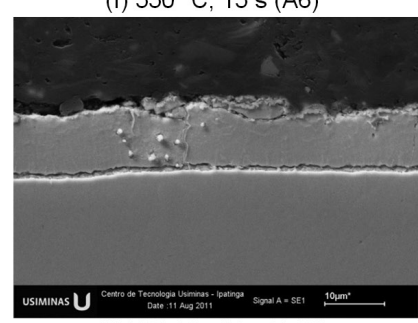

(j) $580^{\circ} \mathrm{C}, 15 \mathrm{~s}(\mathrm{~A} 10)$

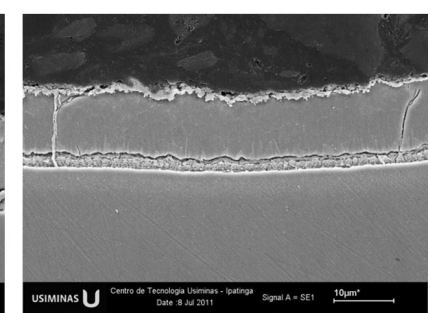

(g) $550^{\circ} \mathrm{C}, 60 \mathrm{~s}(\mathrm{~A} 7)$

Figura I. Microestruturas das seções dos revestimentos GA processados com diferentes tempos e temperaturas de tratamento de galvannealing. 
Os resultados das análises de GDOES para os revestimentos são mostrados nas Figuras 2 a 5. Para cada condição de tratamento térmico de galvannealing são mostrados dois gráficos com os perfis de composição química. $O$ primeiro com as curvas de concentração de $\mathrm{Fe}, \mathrm{Zn}$ e Al e, o segundo mostrando em detalhe a distribuição de $\mathrm{Fe}$ a $\mathrm{Al}$ ao longo do revestimento. No segundo gráfico estão indicadas as faixas de concentração relativas às fases $\eta(<1 \% \mathrm{Fe}), \zeta(5,8-6,5 \% \mathrm{Fe}), \delta(7,3-\mathrm{II}, 7 \% \mathrm{Fe})$, $\Gamma_{\text {। }}(16,3-18,5 \% \mathrm{Fe})$ e $\Gamma(22,2-28,2 \% \mathrm{Fe})$. Para melhor clareza, as fases $\Gamma_{1}$ e $\Gamma$ foram consideradas juntas e identificadas nas figuras apenas por $\Gamma$, correspondendo à faixa de concentração de $16,3-28,2 \% \mathrm{Fe}$. Os valores de concentração de $\mathrm{Al}$ foram multiplicados 100 vezes para permitir uma melhor visualização das curvas. Os perfis de concentração química foram agrupados por tempo de

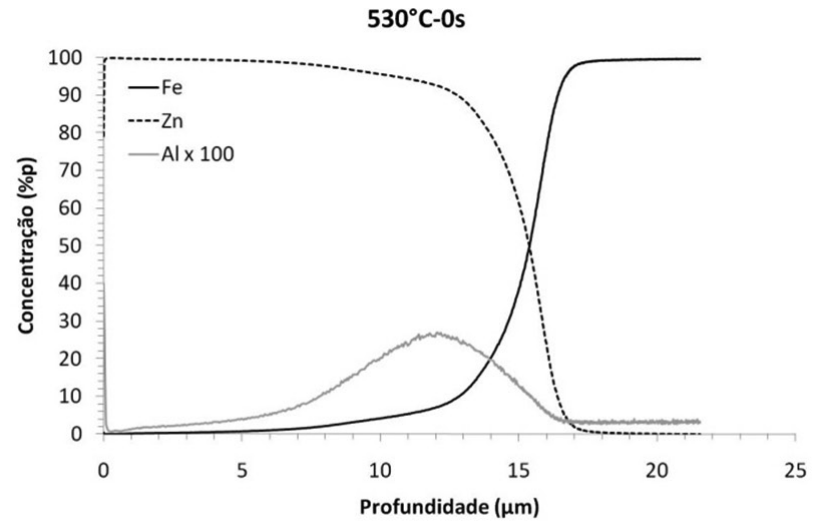

(a)

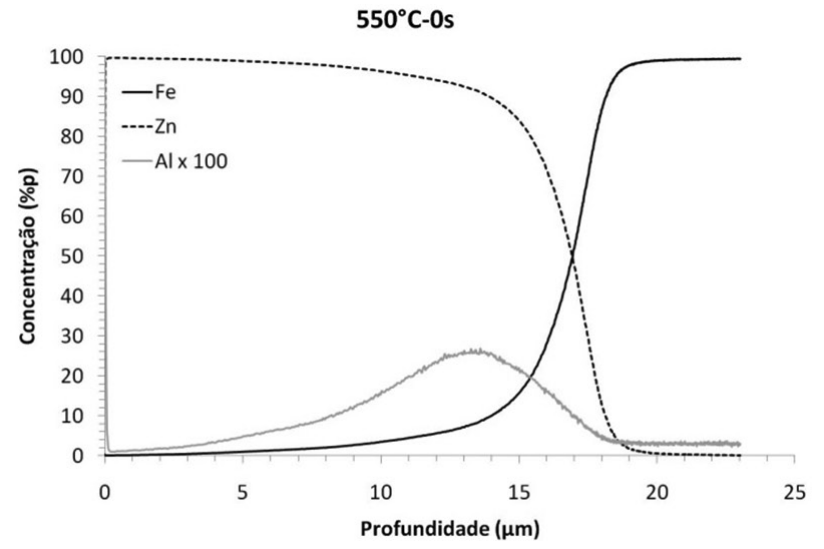

(c)

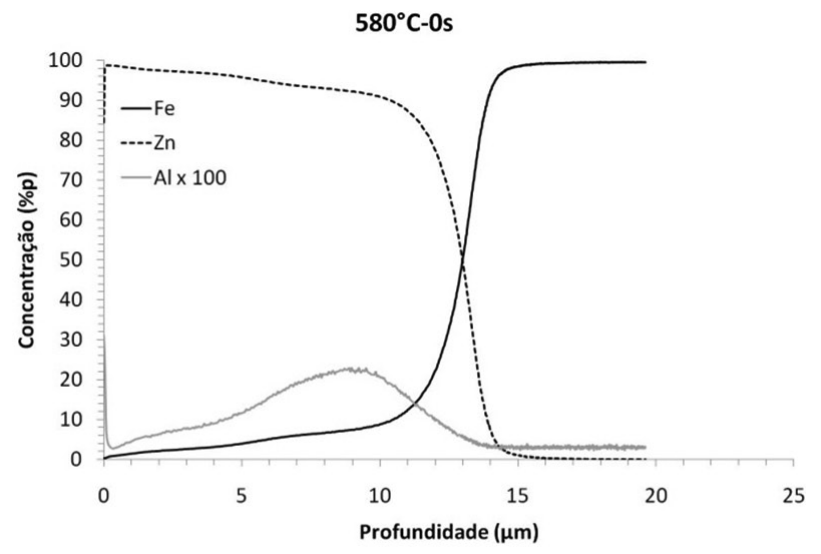

(e)

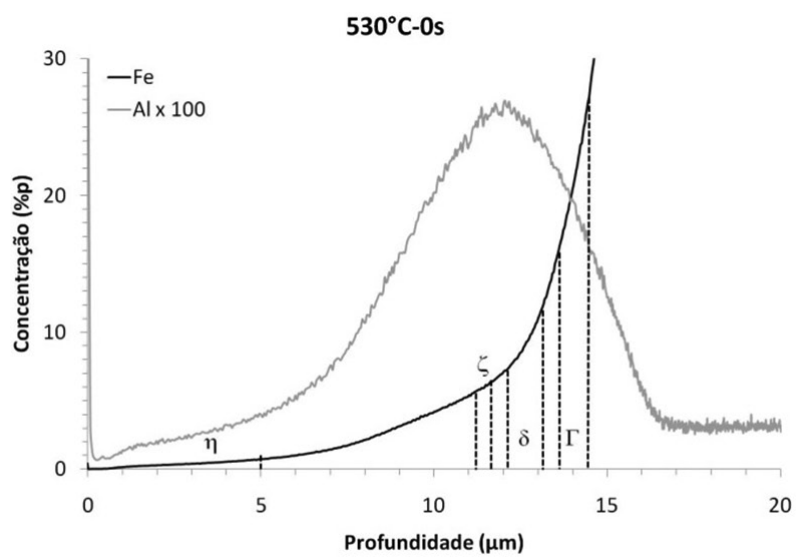

(b)

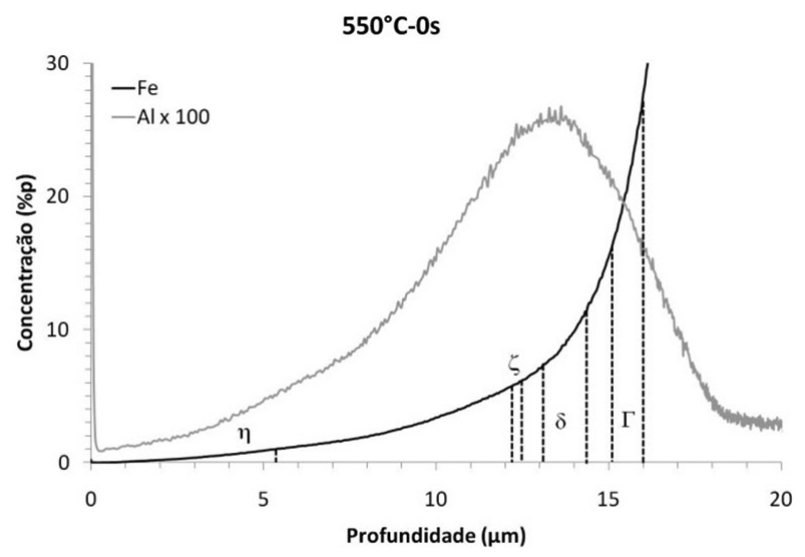

(d)

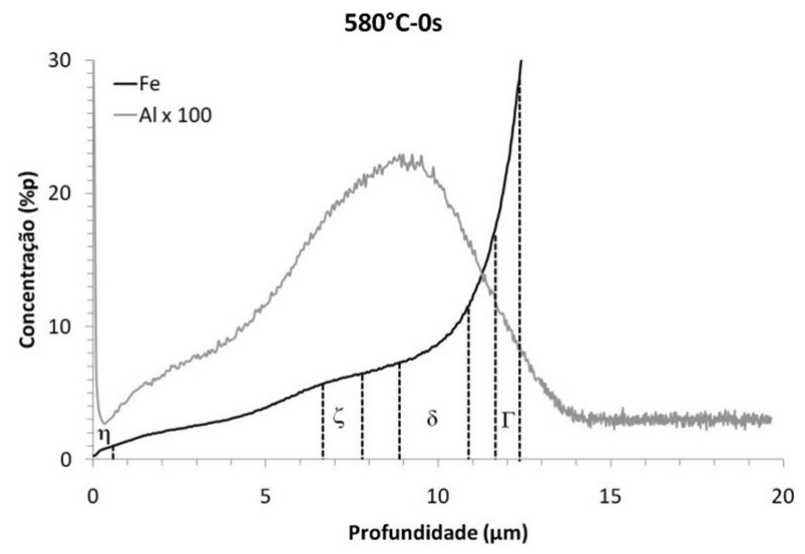

(f)

Figura 2. Perfil de composição química de $\mathrm{Fe}, \mathrm{Al}$ e $\mathrm{Zn}$ para tratamento térmico de galvannealing sem encharque. 
tratamento térmico, de forma a se avaliar a evolução do processo de formação de fases Fe-Zn para as temperaturas de $530^{\circ} \mathrm{C}, 550^{\circ} \mathrm{C}$ e $580^{\circ} \mathrm{C}$.

A partir de análises por GDOES é possível estimar a espessura de revestimentos. A norma ISO 16962:2005(6) estabelece a espessura do revestimento galvanizado como sendo a profundidade na qual a concentração de Zn é igual a $50 \%$ do valor da concentração máxima deste elemento medida entre o início da análise até o teor de $\mathrm{Zn}$ igual a $84 \%$. Neste estudo, foi utilizada uma aproximação da espessura do revestimento para a profundidade na qual ocorre a interseção entre as curvas de concentração de $\mathrm{Zn}$ e Fe, i.e., na concentração de Zn igual a $50 \%$.

Para os revestimentos processados sem encharque $(t=0 \mathrm{~s})$, os perfis de concentração de $\mathrm{Zn}, \mathrm{Fe}$ e Al apresentaram comportamentos similares em todas as temperaturas $530^{\circ} \mathrm{C}-5 \mathrm{~s}$

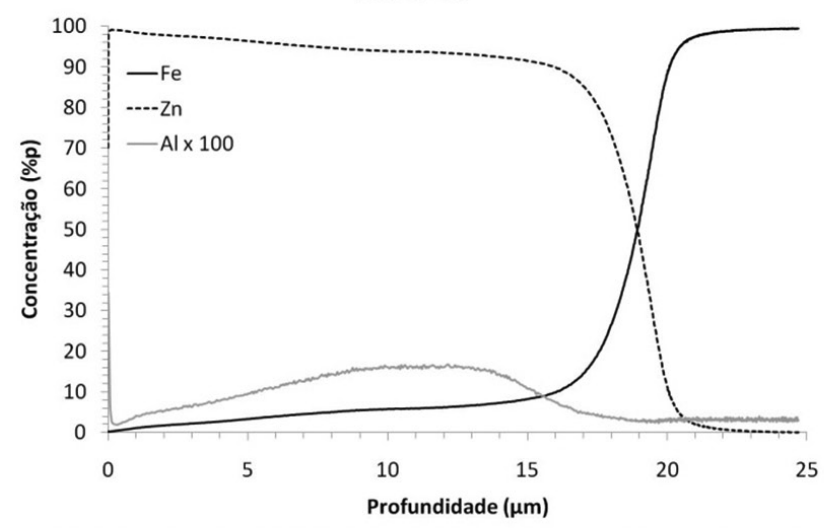

(a)

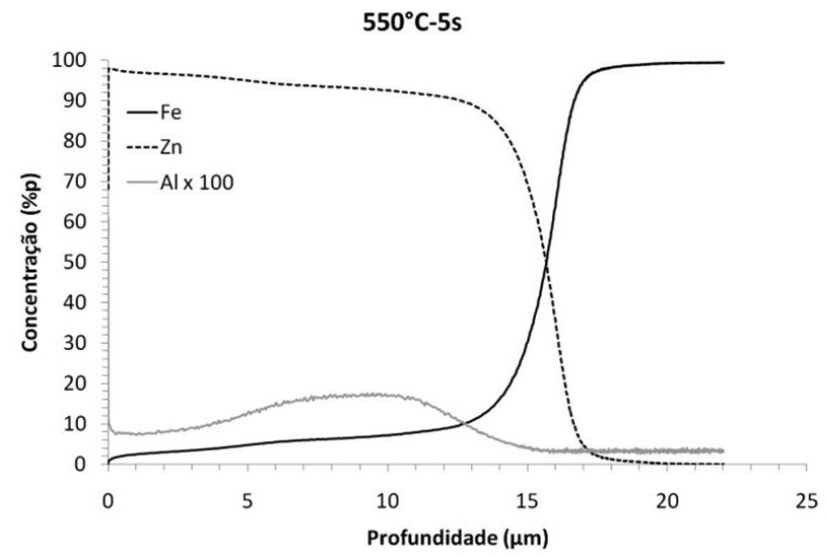

(c)

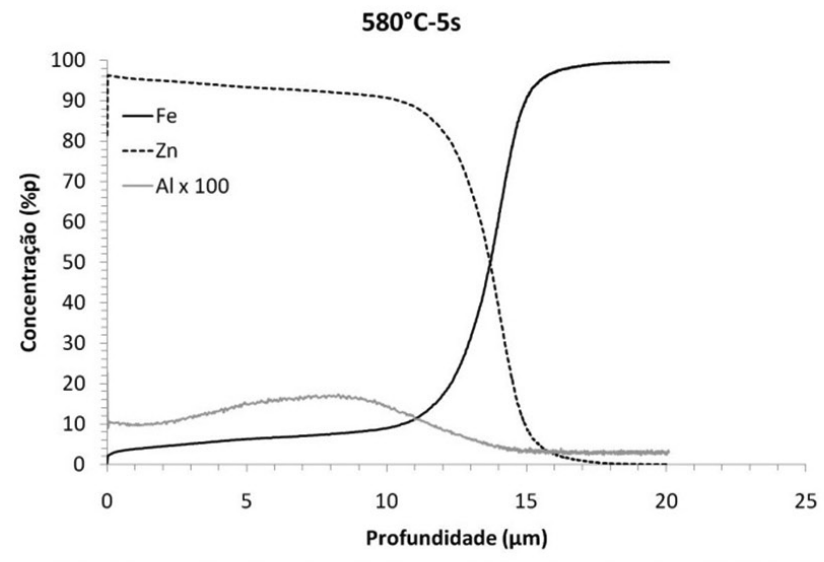

(e)

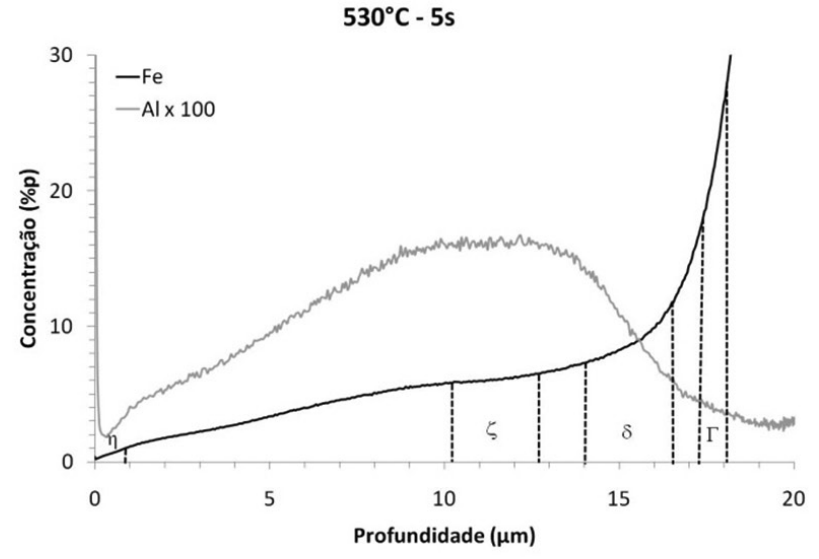

(b)

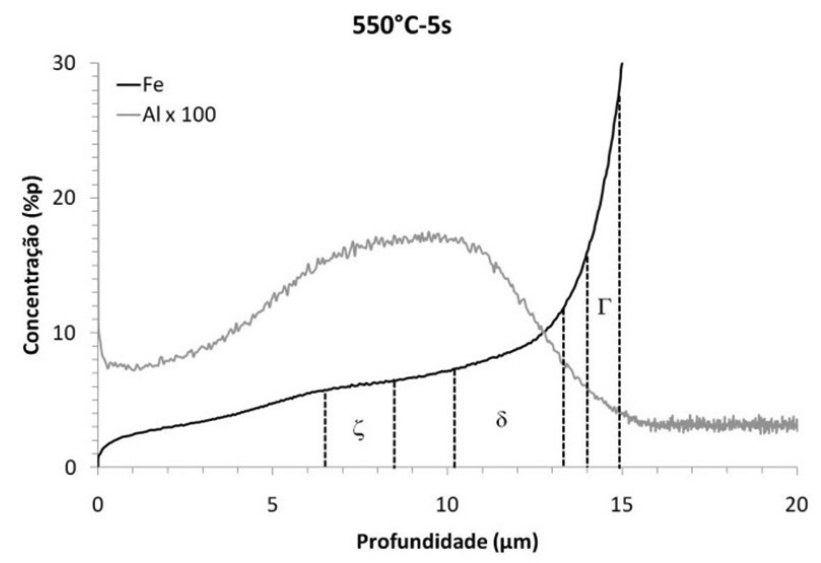

(d)

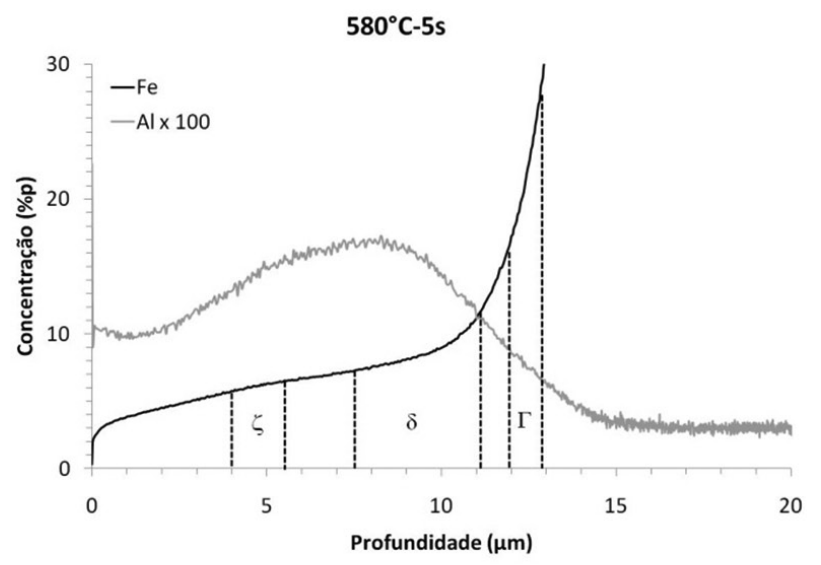

(f)

Figura 3. Perfil de composição química de $\mathrm{Fe}, \mathrm{Al}$ e Zn para tempo de tratamento térmico de galvannealing de $5 \mathrm{~s}$. 
(Figura 2). Observou-se que o teor de $\mathrm{Zn}$ permaneceu próximo a $100 \%$ até cerca de metade da espessura dos revestimentos, quando se observou o início da diminuição da concentração de $\mathrm{Zn}$ e $\circ$ aumento das concentrações de $\mathrm{Fe}$ e Al. $\mathrm{O}$ alumínio se concentrou próximo à interface revestimento/substrato, o que era esperado para este tempo de encharque, uma vez que a presença de $\mathrm{Al}$ está associada à camada inibidora Fe-Al formada na interface durante a passagem da tira de aço pelo pote de $\mathrm{Zn}$ (Figuras 2a, 2c e 2e). Avaliando-se as curvas de distribuição de Fe e Al nos revestimentos (Figuras 2b, 2d e 2f) observou-se que $o$ aumento da temperatura de tratamento térmico aumentou a concentração de $\mathrm{Fe}$ ao longo do revestimento, favorecendo a formação das fases $\mathrm{Fe}-\mathrm{Zn}$, principalmente na $530^{\circ} \mathrm{C}-15 \mathrm{~s}$

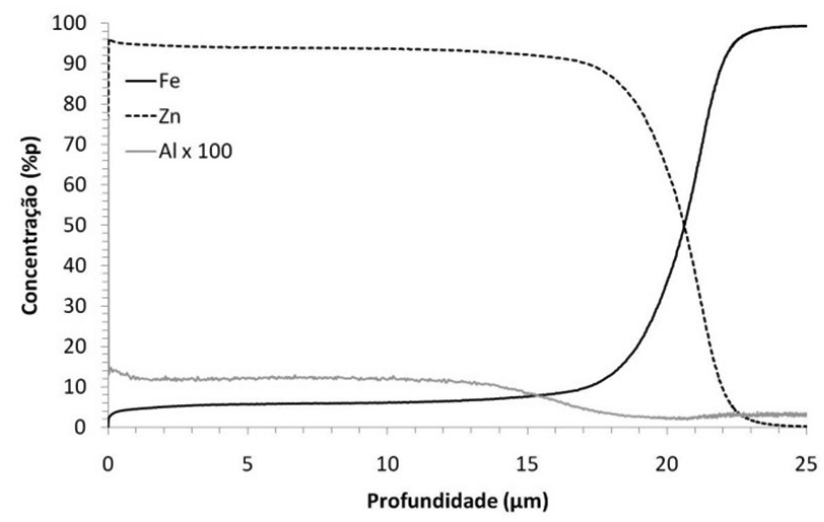

(a)

$550^{\circ} \mathrm{C}-15 \mathrm{~s}$

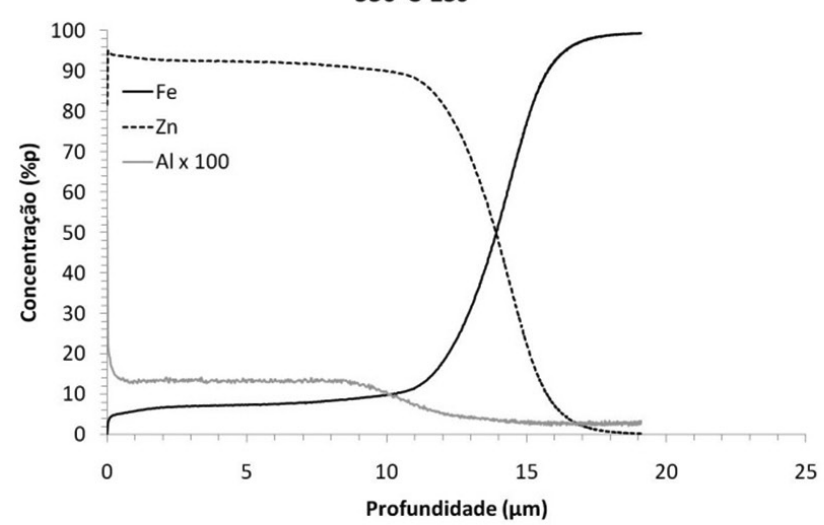

(c)

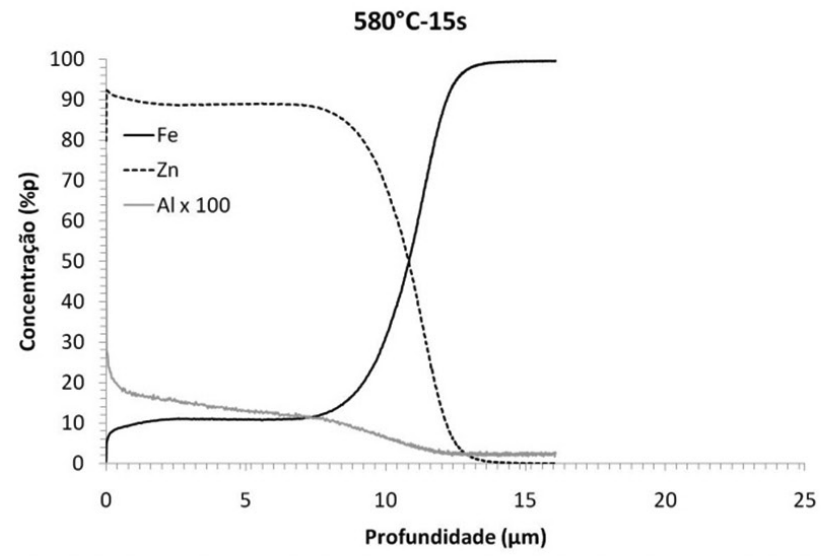

(e)

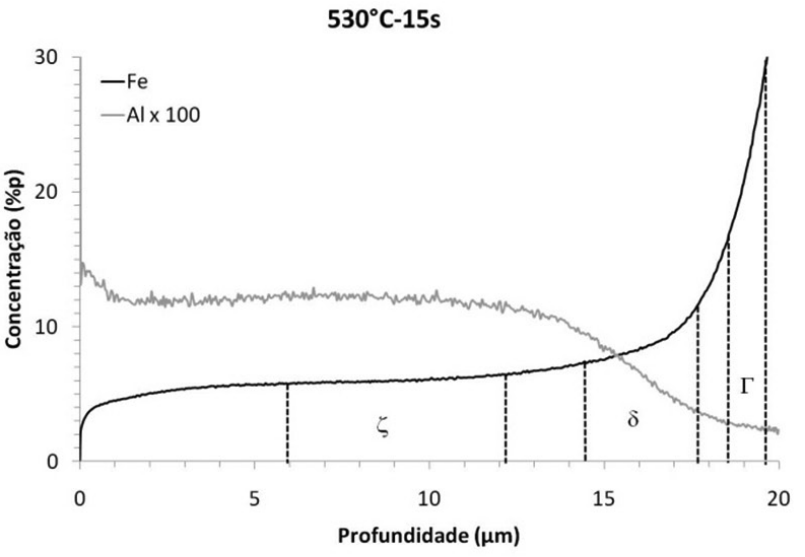

(b)

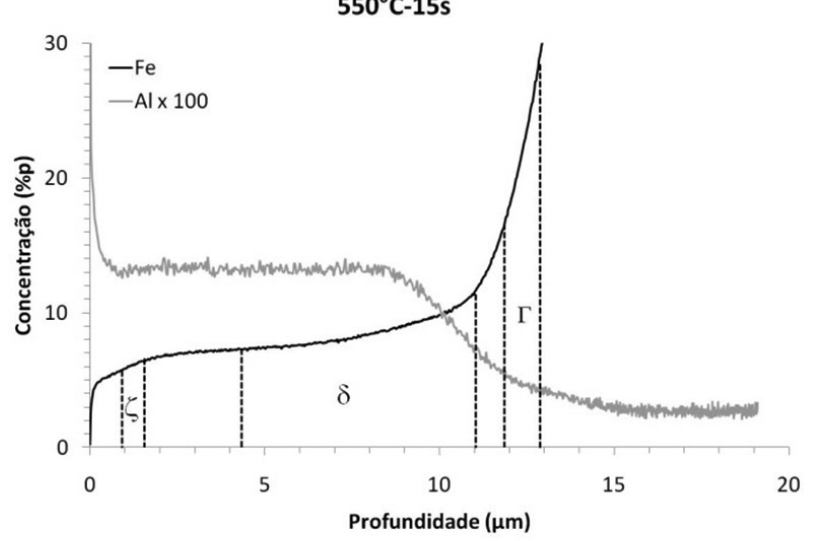

(d)

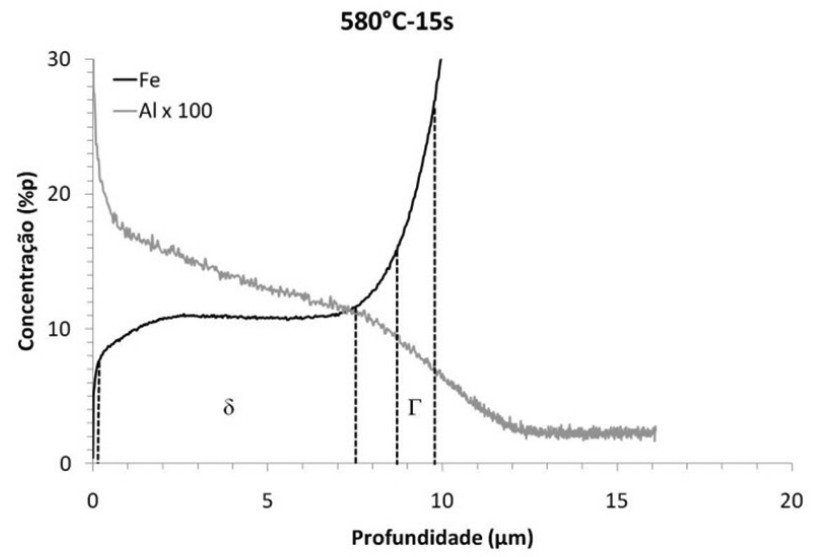

(f)

Figura 4. Perfil de composição química de $\mathrm{Fe}, \mathrm{Al}$ e Zn para tempo de tratamento térmico de galvannealing de $15 \mathrm{~s}$. 
temperatura de $580^{\circ} \mathrm{C}$. Todos os revestimentos apresentaram predominância das fases $\eta$ e $\zeta$. Quanto à distribuição de $\mathrm{Al}$, as maiores concentrações desse elemento foram observadas nas regióes das fases $\zeta$ e $\delta$.

Para o tempo de encharque de 5 segundos, os perfis de concentração de $\mathrm{Zn}$, Fe e Al também apresentaram comportamentos similares em todas as temperaturas (Figura 3). Observou-se que a concentração de Zn permaneceu próxima a $100 \%$ apenas na região mais próxima a superfície, até cerca de um terço da espessura. Após essa profundidade, observou-se o aumento das concentrações de $\mathrm{Fe}$ e $\mathrm{Al}$ (Figuras 3a, 3c e 3e). As curvas de distribuição de Fe e Al (Figuras 3b, 3d e 3f) mostraram o aumento de concentração do Fe ao longo dos revestimentos e a ampliação das regiões com teores de ferro relativos às fases $\zeta$ e $\delta$, em detrimento da região de fase $\eta$, que apareceu isoladamente apenas na temperatura de $530^{\circ} \mathrm{C}$. Nesse tempo de tratamento térmico, as fases $\zeta$ e $\delta$ predominaram nos revestimentos e a região de fase $\Gamma$ aumentou em comparação com os revestimentos processados sem encharque. As curvas de distribuição de Al mostraram a difusão desse elemento no revestimento em direção à superfície, acompanhando a evolução das fases $\zeta$ e $\delta$.

Para o tempo de encharque de $15 \mathrm{~s}$, a influência da temperatura foi mais significativa e os perfis de concentração de $\mathrm{Zn}, \mathrm{Fe}$ e Al, apesar de mostrarem algumas tendências similares, apresentaram comportamentos distintos (Figura 4). Os teores de Zn permaneceram praticamente constantes ao longo da espessura dos revestimentos, sendo de aproximadamente $94 \%$ para temperatura de $530^{\circ} \mathrm{C}, 92 \%$ para $550^{\circ} \mathrm{C}$ e $89 \%$ para $580^{\circ} \mathrm{C}$. Os perfis de concentração de $\mathrm{Fe}$ acompanharam o comportamento dos perfis de $\mathrm{Zn}$, apresentando teores aproximadamente constantes na maior parte da espessura dos revestimentos, sendo de aproximadamente $6 \%$ para temperatura de $530^{\circ} \mathrm{C}, 8 \%$ para $550^{\circ} \mathrm{C}$ e $11 \%$ para $580^{\circ} \mathrm{C}$. A distribuição de alumínio variou com a temperatura, permanecendo praticamente constante ao longo dos revestimentos processados a $530^{\circ} \mathrm{C}$ e $550^{\circ} \mathrm{C}$ e apresentando uma maior concentração próxima a superfície para a temperatura de $580^{\circ} \mathrm{C}$ (Figuras 4a, 4c e 4e).

Avaliando-se as curvas de distribuição de $\mathrm{Fe}$ e Al, para a temperatura de $530^{\circ} \mathrm{C}$ (Figura $4 \mathrm{~b}$ ), observou-se próximo a superfície uma região de coexistência das fases $\eta$ e $\zeta$. Nessa temperatura houve a predominância das fases $\zeta$ e $\delta$, com distribuição uniforme de alumínio nessas fases. Para temperatura de $550^{\circ} \mathrm{C}$, a região de coexistência das fases $\eta$ e $\zeta$ e a região de fase $\zeta$ diminuíram significativamente em comparação com a temperatura de $530^{\circ} \mathrm{C}$. Observou-se a predominância da fase $\delta$ e $\circ$ aumento da contribuição da fase $\Gamma$ (Figura 4d). A distribuição de alumínio permaneceu uniforme e associada às regiões de fases $\zeta$ e $\delta$. Para a temperatura de $580^{\circ} \mathrm{C}$ e tempo de encharque $15 \mathrm{~s}$, a curva de distribuição de $\mathrm{Fe}$ indicou a formação apenas das fases $\delta$ e $\Gamma$, ricas em Fe. A curva de distribuição de $\mathrm{Al}$ mostrou uma maior concentração desse elemento na superfície.

Para o tempo de encharque de 60 segundos na temperatura de $550^{\circ} \mathrm{C}$ (Figura $5 \mathrm{a}$ ), os perfis de $\mathrm{Zn}$, Fe e Al apresentaram comportamentos similares aos observados para os revestimentos processados a temperatura de $580^{\circ} \mathrm{C}$. A concentração de $\mathrm{Zn}$ permaneceu estável ao longo da maior parte da espessura, em média $90 \% \mathrm{Zn}$, bem como, o teor de ferro, em torno de $10 \%$. As análises das curvas de distribuição de $\mathrm{Fe}$ a $\mathrm{Al}$ mostraram que após $60 \mathrm{~s}$ de tratamento térmico o revestimento apresentou basicamente as fases $\delta$ e $\Gamma$, e o alumínio mostrou-se mais concentrado na superfície, permanecendo associado à região de fase $\delta$ (Figura $5 b$ ).

\section{DISCUSSÃO}

A utilização do simulador de processo de galvanização a quente foi fundamental para se produzir amostras adequadas para o estudo da distribuição das fases Fe-Zn $550^{\circ} \mathrm{C}-60 \mathrm{~s}$

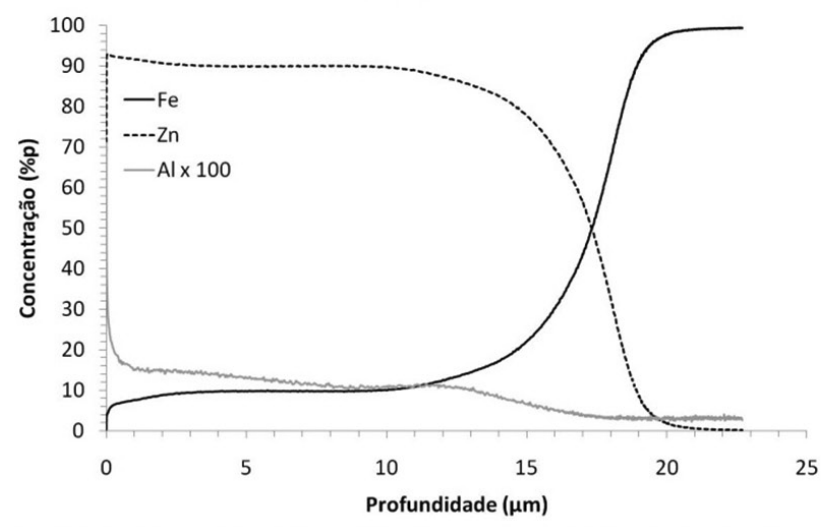

(a)

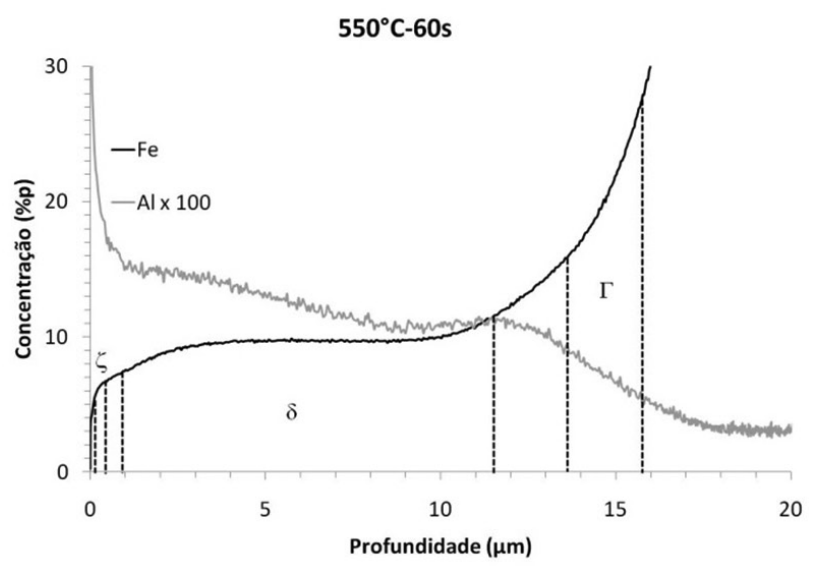

(b)

Figura 5. Perfil de composição química de $\mathrm{Fe}, \mathrm{Al}$ e Zn para tempo de tratamento térmico de galvannealing de $60 \mathrm{~s}$. 
em revestimentos galvannealed. As microestruturas e os perfis de composição química dos revestimentos evidenciaram a influência tanto da temperatura, quando do tempo de encharque do tratamento de galvanneling na formação das fases intermetálicas Fe-Zn.

Os compostos Fe-Zn podem ser diferenciados por seu teor de Fe e essa característica foi utilizada para identificar as fases $\eta, \zeta, \delta$ e $\Gamma$ a partir dos resultados de GDOES. Comparando-se os resultados de MEV/DRX e GDOES pôde-se observar que os perfis de concentração de Fe apresentaram uma boa correspondência com a microestrutura dos revestimentos e com as fases cristalinas identificadas. Por exemplo, para os revestimentos processados sem encharque (Figuras Ia, Id e Ih) os resultados de GDOES (Figura 2) mostraram que a concentração de Fe permaneceu em valores baixos até cerca de $50 \%$ da espessura da camada, indicando a presença de $Z n$ não transformado nessa região, em concordância com as microestruturas observadas. Com o aumento da temperatura para $580^{\circ} \mathrm{C}$, foi observado o aumento da concentração do Fe ao longo do revestimento (Figura $2 \mathrm{f}$ ) e a ampliação das faixas de relativas às fases mais ricas nesse elemento. Tal mudança no perfil descreveu o mesmo comportamento observado na microestrutura dos revestimentos. A coerência entre os perfis de concentração de Fe obtidos por GDOES e as microestruturas e fases cristalinas foram observadas para todos os revestimentos avaliados.

\section{CONCLUSÃO}

As técnicas de análise microestrutural e difração de raios $X$ são convencionalmente utilizadas para caracterização de revestimentos GA, por sua vez, os perfis de composição química obtidos por GDOES ainda são pouco usados para esse fim. Neste trabalho, foi observada boa concordância entre os posicionamentos das estruturas típicas das fases $\mathrm{Fe}-\mathrm{Zn}$ presentes nos revestimentos e os respectivos perfis de composição química obtidos por GDOES, indicando que esta técnica é aplicável, não somente para a caracterização de revestimentos galvanizados industriais, mas também para o estudo das transformações que ocorrem durante o tratamento térmico de galvannealing. Observou-se também uma boa correlação entre as fases cristalinas identificadas por difração de raios $X$ e as determinadas a partir das concentrações de ferro características das fases Fe-Zn. Assim, a caracterização dos revestimentos galvannealed a partir do estudo dos perfis de concentração de Fe e Al obtidos por GDOES mostrou ser uma técnica de grande potencial para a verificação da distribuição qualitativa das fases Fe-Zn.

\section{REFERÊNCIAS}

I Barbosa AHA. Efeito das condições de tratamento de galvanneling na qualidade de revestimento galvanizado a quente "GA", formado sobre aços de alta resistência contendo P e B. 2010, 179p [tese de doutorado]. Belo Horizonte: Universidade Federal de Minas Gerais; 2010.

2 Gellings PJ, Koster D, Kuit J, Fransen T. Synthesis and characterization of homogeneous intermetallic Fe-Zn compounds. Part IV: Thermodynamic properties. Zeitschrift für Metallkunde . 1980;7I:I50-I54.

3 Long JM, Haynes DA, Hodgson PD. Characterization of galvannealed coatings on strip steel. Materials Forum. 2004;27:62-67.

4 Pisonero J, Fernández B, Pereiro R, Bordel N, Sanz-Medei A. Glow-discharge spectrometry for direct analysis of thin and ultra-thin solid films. TrAC Trends in Analytical Chemistry. 2006;25:I I- I8. http://dx.doi.org/10.1016/j. trac.2005.04.019

5 Glow Discharge Dot Com. Glow discharges. 2013 Mar 22 [acesso em 23 abril 20 I 2]. Disponível em: http://www. glow-discharge.com.

6 International Organization for Standardization. ISO 16962-2005. Surface chemical analysis - Analysis of zinc and/or aluminium metallic coatings by glow-discharge optical-emission spectroscopy. Geneva, 2005.

Recebido em: 15/04/2013

Aceito em: 28/06/20I3 